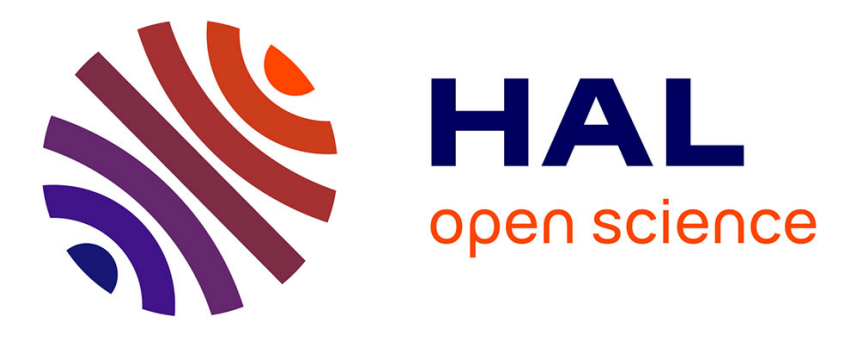

\title{
General Applicability of the Coupling Model to Viscoelasticity of Polymers : from Local Segmental Motion to Terminal Flow
}

K. Ngai, R. Rendell, D. Plazek

\section{- To cite this version:}

K. Ngai, R. Rendell, D. Plazek. General Applicability of the Coupling Model to Viscoelasticity of Polymers: from Local Segmental Motion to Terminal Flow. Journal de Physique IV Proceedings, 1996, 06 (C8), pp.C8-555-C8-566. 10.1051/jp4:19968121 . jpa-00254552

HAL Id: jpa-00254552

https://hal.science/jpa-00254552

Submitted on 1 Jan 1996

HAL is a multi-disciplinary open access archive for the deposit and dissemination of scientific research documents, whether they are published or not. The documents may come from teaching and research institutions in France or abroad, or from public or private research centers.
L'archive ouverte pluridisciplinaire HAL, est destinée au dépôt et à la diffusion de documents scientifiques de niveau recherche, publiés ou non, émanant des établissements d'enseignement et de recherche français ou étrangers, des laboratoires publics ou privés. 


\title{
General Applicability of the Coupling Model to Viscoelasticity of Polymers: from Local Segmental Motion to Terminal Flow
}

\author{
K.L. Ngai, R.W. Rendell and D.J. Plazek* \\ Naval Research Laboratory, Washington, DC 20375-5320, U.S.A. \\ * Department of Materials Science \& Engineering, University of Pittsburgh, Pittsburgh, PA 15361, U.S.A.
}

\begin{abstract}
We survey the important problems in polymer viscoelasticity that impede progress in this field. These problems manifest themselves as spectacular anomalies in the viscoelastic data that have been reproduced in different polymers and in different laboratories, but have largely been put aside for lack of explanation. Without solving these difficult problems, there can be no satisfactory understanding of the viscoelastic properties of polymers spanning across the local segmental motion, the glass-rubber softening dispersion, the rubbery plateau and the terminal dispersion. The coupling model, which has general applicablity to various relaxation mechanisms of polymers and beyond polymers, is able to resolve all these problems.
\end{abstract}

\section{INTRODUCTION}

The coupling model (CM) [1-3] is a general approach to dynamics of constrained or interacting systems, shown to be applicable to many problems in different systems $[4,5]$. Recent versions of the coupling theory are based on classical mechanics for systems that exhibit chaos caused by the anharmonic (nonlinear) nature of the interactions between the basic molecular units [2,3]. For example, intermolecular interactions between monomer units in a polymer modelled by the Lennard-Jones potential are highly anharmonic and give rise to chaotic dynamics [6]. Although a rigorous theory based on first principles is still at large, we are quite confident that the results of the $\mathrm{CM}$ are basically correct. This confidence is gained by (1) the results being borne out by simple models [2,3], (2) the many successes of the $\mathrm{CM}$ in its applications to a variety of challenging problems in several fields [4,5], and (3) the direct verification of the fundamental results by recent experiments. The latter include quasielastic neutron scattering experiments in several polymers including polyvinylchloride [7], polyisoprene and polybutadiene [8], very high frequency dielectric measurements for glassy and non-glassy ionic conductors $[9,11]$ and glass-forming molten salts $[10,11]$, and molecular dynamics simulations of small molecule liquids [12]. The fundamental results of the coupling theory, which have remained unchanged since its inception fifteen years ago [1], are restated here as follows. There exists a temperature insensitive cross-over time, $t_{c}$, before which $\left(t<t_{c}\right)$ the basic units relax independently with correlation function

$$
\phi(t)=\exp \left(-\frac{t}{\tau_{o}}\right)
$$

and afterwards $\left(t>t_{c}\right)$ with the slowed-down stretched exponential function

$$
\phi(t)=\exp \left[-\left(\frac{t}{\tau}\right)^{1-n}\right]
$$

where $n$ is the coupling parameter whose value lies within the range $0 \leq n<1$ and depends on the intermolecular interaction. In polymers such a cross-over has been observed by neutron scattering $[7,8]$ for segmental motion at $t_{c} \cong 2$ ps.

Continuity of the two pieces of the correlation function at $t=t_{c}$ leads to the important relation

$$
\tau^{*}=\left[t_{c}^{-n} \tau_{o}\right]^{\frac{1}{1-n}}
$$

which links the effective (i.e. after cooperative dynamical contraints between the relaxing molecular units have been taken into account) relaxation time, $\tau^{*}$, to their independent (i.e. without taking into account of the 
cooperative dynamical constraints) relaxation time, $\tau_{0}$. It is useful to note that when considering macroscopic relaxation phenomena occuring at long times, the condition $\tau_{d} / t_{c} \gg 1$ holds. As a result, the linear exponential $\exp \left(-t / \tau_{0}\right)$ has decayed by an insignificant amount at $t=t_{c}$, and the correlation function $\phi(t)$ is practically given at all times by the stretched exponential piece. These are general results which are applicable to different interacting systems and relaxing units with length scales as small as the local segmental motion responsible for the glass "transition" and as large as the entire polymer molecule or polymer cluster. The results, as written down in equations 1-3 and specialized to relaxation or diffusion of entire polymer chains, hold only for systems in which all the relaxation species and their $\tau_{\mathrm{o}}$ 's are the same. For the motions of the entire polymer chain, polydispersity (i.e. a distribution of chain lengths) introduces a distribution of the initial relaxation times, $\tau_{0}$ 's, and possibly even a distribution of the coupling parameters, $n$ 's, resulting in a distribution of $\tau^{*}$ 's. These additional complications further broaden the time dependence beyond that given by eq. 3 . The coupling model (CM) has been applied to different zones of viscoelastic response of polymers from some secondary relaxations, local segmental motion, the glass-rubber softening dispersion and the terminal relaxation [1317]. These applications have solved many previously puzzling problems in the different zones. As far as the authors know, no other model or theory has demonstrated such wide applicability to relaxations in polymers. We are certain that no current theory of polymer dynamics, except the CM, is applicable to problems in other systems including the glassy ionic conductors [4]. In this work we shall outline some of the oustanding problems in polymer dynamics in different viscoelastic zones and indicate how the CM has resolved them. Due to space restrictions, the discussion of most problems will be brief and refer to earlier works. The exception is the dynamics of terminal flow which we shall discuss in some detail because the results presented are new.

\section{THE LOCAL SEGMENTAL MOTION (GLASS “TRANSITION")}

In the past, theoretical studies of relaxational properties of glass-forming non-polymeric and polymeric materials, like most of the experiments carried out, were mainly confined to the long or macroscopic time regime (say $\gg 1$ nanosecond). Starting from the days of $\mathrm{R}$. Kohlrausch at Göttingen, nearly 150 years ago [18], much experimental data in the long time regime have been accumulated. The materials studied include a wide variety of molecular, inorganic, ionic, and polymeric glass-forming substances. The glass "transition" is a fundamental problem that remains in the frontier of basic research. Recently, particularly within the past five years, quasielastic neutron scattering (QENS), dynamic depolarized light scattering and high frequency conductivity measurements have opened up our knowledge of short time dynamics [712]. The existence of a fast process (eq.1) at microscopic times $t<t_{\mathrm{c}}$ and the crossover to a slow process (eq.2), now written as exp- $\left(t / \tau_{\alpha}^{*}\right)^{1-n_{\alpha}}$, predicted by the coupling model (CM) was demonstrated to be in good agreement with QENS data of polyvinylchloride and other polymers [7]. Continuity of the correlation function at $t_{\mathrm{c}}$ was observed, validating the relation between $\tau_{\mathrm{o}}$ and $\tau^{*}$ given by eq.3. Subsequently, similar agreement has been found in QENS spectra of polyisoprene and polybutadiene by Zorn et al.[8]. These neutron scattering experiments were performed with $Q$-vectors typically of the order of $Q \approx 1 \AA^{-1}$, which correspond to length scales of the order of $Q^{-1} \approx 1 \AA$. Hence, they probe the local segmental motion (LSM) responsible for the glass "transition" and verify directly the fundamental premise of the CM for this local motion. Ancillary experimental data show that the $Q$ and $T$ (temperature) dependences of $\tau_{\mathrm{o}}$ and $\tau^{*}$ are related according to eq.3. Moreover, the $Q$ and $T$ dependences of $\tau_{\mathrm{o}}$ correspond to that expected of a simple local intramolecular motion of the polymer chain in the absence of intermolecular (interaction) coupling, consistent with the interpretation of the CM.

Mechanical, dielectric and photon correlation spectroscopic data of the LSM performed at long times or low frequencies have found that the correlation function invariably is well approximated by the Kohlrausch function (eq.2). The coupling parameter, $n_{\alpha}$, of the LSM in polymers changes with the chemical structure of the monomer unit. Polymers with chemical structures that have a larger capacity for intermolecular coupling tend to have a larger $n_{\alpha}$ in accord with the physical meaning attached to $n_{\alpha}$ in the 
CM. An individual polymer has its own glass transition temperature, $T_{g}$, which can be defined operationally as the temperature at which its relaxation time $\tau_{\alpha}^{*}\left(T_{g}\right)$ equals an arbitrarily chosen long time, say 100 seconds. The temperature dependences of different polymers can be compared in a single plot of $\log \left[\tau_{\alpha}^{*}(T)\right]$ against a scaled reciprocal temperature $T_{g} / T$. In this plot [19], the individual curves of different polymers exhibit a pattern in which a polymer having a larger $n_{\alpha}$ has a steeper dependence of $\tau_{\alpha}^{*}$ on $T_{g} / T$. This pattern is expected by the predictions of the CM [19]. For example, polystyrene (PS) has a bulkier phenyl ring attached to a carbon in the main chain while polyisobutylene (PIB) has a more compact symmetric backbone. The CM expects that $n_{\alpha}$ of PS is larger than that of PIB. Experimentally, $n_{\alpha}(\mathrm{PS})=0.64$ and $n_{\alpha}(\mathrm{PIB})=0.45$, and $\tau_{\alpha}^{*}$ of PS has a stronger $T_{g} / T$-dependence than PIB.

Considering that the results of the $\mathrm{CM}$ are in agreement with both the short time and the long time dynamics, we are justified to conclude that a significant part of the physics of the glass "transition" of polymers has been captured.

\section{THE GLASS-RUBBER SOFTENING DISPERSION OF ENTANGLED POLYMERS}

For a high molecular weight polymer in which the chains are sufficiently long for them to entangle, the viscoelastic response measured in shear compliance $J(t)$ that rises from the glassy compliance $J_{\mathrm{g}} \approx 10^{-9} \mathrm{~Pa}^{-1}$ (glassy modulus $G_{\mathrm{g}} \approx 10^{9} \mathrm{~Pa}$ ) and ends at the beginning of the rubbery entanglement plateau with $J_{\mathrm{N}} \approx 10^{-6}$ $\mathrm{Pa}^{-1}\left(G_{\mathrm{N}} \approx 10^{6} \mathrm{~Pa}\right)$ is referred to as the glass-rubber softening dispersion. The initial rise is due to the LSM discussed in the previous section. It turns out that the LSM can account only for an initial increase of $J(t)$ up to $J_{\text {ed }}$ about 4 to 5 times $J_{g}$, depending on the polymer $[5,20,21]$. The rest of the increase in $J(t)$ is generally recognized to be due to the sub-Rouse modes [27] and the Rouse modes (R) generalized for undiluted polymers [22]. The Rouse modes are normal modes of a chain that are viewed as consisting of a sequence of Gaussian submolecules. In spite of it being oversimplified, the Rouse model plays an important role in polymer dynamics. The width of the softening dispersion, determined by the separation between the logarithm of the relaxation times $\tau_{\alpha}^{*}$ and $\tau_{\mathrm{R}}$ of the LSM and the Rouse modes respectively, varies greatly from one polymer to another. It is narrow for a polymer with a larger $n_{\alpha}$ such as PS but much broader for a polymer with a smaller $n_{\alpha}$ such as PIB. The viscoelastic data of PS in the glass-rubber transition is clearly not thermorheologically simple (TS) [5,23-25]. The breakdown of TS is caused by the fact that $\tau_{\alpha}^{*}$ increases much more rapidly with decreasing temperature than $\tau_{R}$ of the Rouse modes. The degree of breakdown of TS being higher in PS than in PIB [5,26,27] originates from the difference in the temperature dependences of $\tau_{\alpha}^{*}$ and $\tau_{\mathrm{R}}$ being more spectacular in PS than in PIB. The CM explains these facts by applying eq. 3 to the LSM and the Rouse modes. While both modes, $\alpha$ and $R$ have the same friction factor, $\zeta_{\mathrm{o}}(T)$, for their independent relaxation times, $\tau_{\mathrm{o} \alpha}$ and $\tau_{\mathrm{oR}}$, appearing in eq.1, their effective relaxation times have different friction factors

$$
\tau_{\alpha}^{*} \propto\left(\zeta_{o}(T)\right)^{1 /\left(1-n_{\alpha}\right)} \quad \text { and } \quad \tau_{\mathrm{oR}} \propto \zeta_{0}(T)
$$

respectively. The latter result is based on the fact that Rouse modes in the softening dispersion are not subjected to entanglement interactions and their coupling parameters have zero values. We have shown earlier that this result readily explains the breakdown of TS observed in the softening dispersion [4,5]. A polymer with larger $n_{\alpha}$ will witness its $\tau_{\alpha}^{*}$ encroaching more towards the longer $\tau_{\mathrm{oR}}$ as temperature falls, resulting in a narrower width of the observed softening dispersion. On the other hand, the encroachment of $\tau_{\alpha}^{*}$ is less for polymers with smaller $n_{\alpha}$ and consequently it has a broader softening dispersion. We know of no other model or theory that provides explanations of these experimental data that have been duplicated in several laboratories [23-25]. 


\section{VISCOELASTIC ANOMALIES IN LOW MOLECULAR WEIGHT POLYMERS}

It is well known that the steady state recoverable compliance, $J_{e}^{0}$, of unentangled polymers obtained at temperatures sufficiently high above the glass transition temperature is remarkably well described by the modified Rouse model prediction: $J_{e}^{0}=0.40(M / \rho R T)$, where $M$ is the molecular weight and $\rho$ the density. However, as the temperature is decreased towards $T_{\mathrm{g}}$, a spectacular viscoelastic phenomenon was observed in the recoverable compliance $J_{\mathrm{r}}(t)$. The steady state recoverable compliance $J_{e}^{o}$ decreases many fold. The effect grows with lower molecular weights. For example, in PS with $M=3400 J_{e}^{o}$ drops by thirty times the Rouse value as $T_{\mathrm{g}}$ is reached [21]. This effect is found in other polymers including polypropylene glycol (PPG) [28,29] and polymethylphenylsiloxane [21,29] and hence is general. The relaxation time $\tau_{\alpha}^{*}$ of the LSM has a much stronger $T$-dependence than that of the Rouse time $\tau_{\mathrm{R}}$ measured by the product $\eta_{e}^{o}$ where $\eta$ is the viscosity. Dielectric relaxation data of PPG [29] have confirmed these findings. Only a few textbooks including the classic by Ferry [22] mentioned this effect. Such a dramatic viscoelastic effect discovered a long time ago has only been rationalized by the CM [21,28-30]. Again, the fundamental cause lies in the difference between $\tau_{\alpha}^{*}$ and $\tau_{R}$ (see Equation 4 ). The difference, and hence the effect, is reduced for polymers with a smaller coupling parameter $n_{\alpha}$ such as PIB. This expectation is borne out by experimental measurements of PS and PIB with the same number of carbon atoms in the polymer backbones [30].

\section{COUPLING MODEL FOR TERMINAL RELAXATION OF ENTANGLED POLYMERS}

The application of the coupling model to the terminal relaxation was started with the publication of References 13 and 14. Although a few publications follow [15-17], no serious effort was made thereafter to improve the initial theoretical considerations which starts with the Rouse modes $p=1,2,3, \ldots$ of a chain and slows down their relaxation, $\varphi_{p}(t)=\exp -\left(t / \tau_{p}\right)$, by the entanglement interaction between chains to a stretched exponential relaxation, $\varphi_{p}(t)=\exp -\left(t / \tau_{p}^{*}\right)^{1-n_{p}}$ for $t>\hat{t}_{c}$. The relaxation times, $\tau_{p}$, of the Rouse modes are given by $\tau_{p}=\zeta(T) N^{2} b^{2} /\left(3 \pi^{2} k_{B} T\right) p^{2} \propto \zeta_{o}(T) M^{2} / p^{2}$. Here $\zeta$ is the friction of each bead connecting $N$ identical segments each with mean square length equal to $b^{2}$, $\zeta_{o}$ is the monomeric friction coefficient which is related to $\zeta$ by $\zeta_{\alpha} P=\zeta N$ where $P$ is the degree of polymerization, and the molecular weight $M$ is proportional to $N$. The entanglement coupling parameter, $n_{p}$, is equal to zero for modes whose wavelengths are shorter than than the entanglement distance. Such a crossover from Rouse dynamics to slowed-down dynamics was observed in Monte Carlo [31,32] and molecular dynamics simulations [33]. Since the slowing down of the Rouse modes by the entanglement interaction involves a longer length scale compared with intermolecular interactions for LSM, we expect $\hat{t}_{c}$ of entanglement coupling to be significantly longer than $t_{c}$ for coupling of LSM.

The continuity requirement of the correlation function $\varphi_{p}(t)$, given in two pieces by eqs 1 and 2 at $t=\hat{t}_{c}$, gives rise to the important relation $\tau_{p}^{*}=\left(\hat{t}_{c}^{-n_{p}} \tau_{p}\right)^{1 /\left(1-n_{p}\right)}$. Substituting the expression of $\tau_{p}$ for $\tau_{o}$ into eq 3 , the following dependencies on $M$ and $T$ are obtained

$$
\tau_{p}^{*} \propto\left(\zeta_{o}(T) M^{2}\right)^{1 /\left(1-n_{p}\right)}
$$

In the original formulation [13-17], only the $p=1$ Rouse mode with the longest relaxation time, $\tau_{1}$, often referred to as the Rouse relaxation time, $\tau_{R}$, was considered. For a choice of the coupling parameter, $n_{1}$, in the range $0.40<n_{1}<0.45$, eq. 5 shows that $\tau_{p}^{*} \propto M^{2 /\left(1-n_{1}\right)}$ has the molecular weight exponent in the range $0.33<\left[2 /\left(1-n_{1}\right)\right]<0.37$ found by rheological experiments. Only a limited amount of rheological data on monodisperse polymer melts was available in 1982. The frequency dependence of the loss modulus, $G$ " $(\omega)$, calculated by Fourier transformation of the shear modulus,

$$
G(t)=G_{N}^{0} \exp -\left(t / \tau^{*}\right)^{1-n_{1}},
$$


(which has time dependence as $\phi(t)$ given by eq 2) was used to fit the hydrogenated polybutadiene (HPB) data of Raju et al. [34]. Good agreement with experimental data of a $1.84 \times 10^{5}$ molecular weight HPB sample was obtained with $n_{1}=0.40$. Such a value of $n_{1}$ in eq 6 leads to the product $J_{e}^{0} G_{N}^{0}=2.04$ which falls into the acceptable range found experimentally. The temperature dependence of $\tau^{*}$ in HPB and PE is Arrhenius with activation enthalpy, $E_{a}^{*}$. According to eq 5, the Rouse monomeric friction coefficient $\zeta_{0}(T)$ has an activation enthalpy, $E_{a}=\left(1-n_{1}\right) E_{a}^{*}$.The values of $E_{a}$ obtained for HPB and PE are $4.2 \mathrm{kcal} / \mathrm{mol}$ and $3.6 \mathrm{kcal} / \mathrm{mol}$ respectively, which can be identified with the chain conformation energy barriers in these polymers. These results are supported by self diffusion data in the same polymers [16].

In spite of these encouraging developments, some of which are cited above, no further work was done to improve the model. The structure of the model was kept in its simplest form. In the past ten years, a plethora of experimental measurements of the terminal relaxation of monodisperse polymers have emerged to made its frequency dependence clear [35]. It has become evident that the low frequency side of the measured $G$ " $(\omega)$ peak is narrower than is predicted by eq 6 with $n_{1} \geq 0.40$. Dielectric normal mode relaxation measurements on high cis content polyisoprene (PI) have revealed [36-40] that the dielectric loss, $\varepsilon^{\prime \prime}(\omega)$, peak starting approximately one decade to the right of the peak frequency and down to the low frequencies is significantly narrower than originally predicted [13-17]. However, the high frequency side of the $\varepsilon^{\prime \prime}(\omega)$ peak starting at approximately one decade higher than the peak frequency behaves like $\varepsilon^{\prime \prime}(\omega) \propto \omega^{-\alpha}$ with $\alpha \cong 0.33-0.30$ is sufficiently dispersive to be consistent with $n_{p} \approx 0.40$ to 0.45 , provided contributions from the odd-number modes $(p=1,3,5, \ldots)$ are all included. At this time it is clear that the original version of the $\mathrm{CM}$ for terminal relaxation is inadequate. The purpose of this work is to locate the inadequacy of the original model, fix it by modifying the model to be consistent with the frequency dependence of the terminal relaxation, and show it is consistent with dielectric relaxation data of dilute probe PI chains in a matrix formed by very high molecular weight polybutadiene (PB) chains $[41,42]$ or in loosely cross-linked PB networks [43].

It was generally recognized by various workers that the topological constraints imposed by other chains in an entangled linear polymer melt are primarily lateral, running parallel along the chain contour [44-46], even before the reptation mechanism of DeGennes [47] and the tube/reptation model of Doi and Edwards [48] were proposed. The lateral constraints retard sustained motions transverse to the chain contour. Hence, each chain acts as if it were confined in a tube. The existence of a tube or lateral constraints (LC) restricting the free Rousean motion of a chain was confirmed by experiment [49] and should be incorporated into any theory of chain motion. Nevertheless, the actual manner in which the chains move is . still an open question. Certainly, reptation is a clear option but is likely to be the one that is most inefficient. This is because in resorting to reptation one has given up the consideration of other possible options in favor of the tortuous diffusion of the chain along its own path. This is reflected in the predicted zero shear viscosity, $\eta$, being too large and the recoverable compliance, $J_{e}^{0}$, being too small compared with experimental observations [50].

An alternate mechanism of chain motion is the constraint dynamics of entangled chains that follows from the general physical principle of the CM, i.e. crossover at $t=\hat{t}_{c}$ from relaxation of Rouse- modes-in-a-tube to a slowed down relaxation. In earlier works [13-17], the effects due to the presence of LC had not been included explicitly into the formulation based on slowed down isotropic Rouse modes (see Eq.6). However, $L C$ were implicitly assumed because the plateau modulus, $G_{N}^{0}$, was taken to be independent of molecular weight which is appropriate for an entangled linear chain liquid. Now, let us incorporate the LC explicitly into the consideration of the CM. We hasten to clarify that the basic mechanism of the CM continues to hold. The only change is to take into consideration the additional complication that arises when LC is included. We limit our discussion in this paper to dielectric normal mode relaxation of polymers whose monomer has a dipole moment parallel to the chain direction.

Starting at $t=\hat{t}_{c}$, the LC have the effect of slowing down the relaxation rate, $\tau_{p}^{-1}$, of the $p$-th Rouse-mode-in-a-tube $\left(p>p_{\mathrm{e}}=N / N_{\mathrm{e}}\right)$. According to the coupling model, the Rouse rate $\tau_{p}^{-1}$ is slowed 
down increasingly with time as $\tau_{p}^{-1}\left(\hat{\omega}_{c} t\right)^{-n_{p}}$. Here $\hat{\omega}_{c}$ is related to $\hat{t}_{c}$ by the relation: $\hat{\omega}_{c}=\left[1-n_{p}\right]^{-1 / n_{p}} \hat{t}_{c}^{-1}[51]$. The coupling parameter, $n_{p}$, remains constant as long as the LC remain unchanged as time increases. The LC are provided by the surrounding chains whose motions cannot be ignored. The LC are mitigated as soon as neighboring chains that supply the LC move away. Therefore, the $\mathrm{LC}$ are not permanent as assumed in the previous works where $n_{p}$ in eq 2 was treated as a constant for all times $t>\hat{t}_{c}$. Instead, the LC have finite lifetimes, $\tau_{L C M}$, which should be of the order of $\tau_{p}^{*}$. The end-to-end vector correlation function with the slowed-down Rouse-modes-in-a-tube from eq 2, acting as if the latter holds for all times with constant $n_{p}$, is given by [36]

$$
\langle r(0) \cdot r(t)>|\left\langle r^{2}\right\rangle \equiv g(t)=\left(8 / \pi^{2}\right) \sum_{p, o d d}\left(1 / p^{2}\right) \exp \left(-\left(t / \tau_{p}^{*}\right)^{1-n_{p}}\right)
$$

We can get an estimate of the lifetime of the $\mathrm{LC}$ or the lateral constraints mitigation time, $\tau_{L C M}$, from

$$
\tau_{L C M}=\int_{0}^{\infty} g(t) d t=\left(\frac{8}{\pi^{2}}\right) \sum_{p, o d d}\left(1 / p^{2}\right)^{\left(1+\frac{1}{1-n_{p}}\right)}\left\{\Gamma\left(1 /\left(1-n_{p}\right)\right) /\left(1-n_{p}\right)\right\} \tau_{1}^{*},
$$

which can be obtained numerically once the $n_{p}$ 's are specified. If there were $z$ neighboring chains that define the LC and they are mitigated by the reorientation of any one of the $z$ chains, $\tau_{L C M}$ is then given by

$$
\tau_{L C M}=\int_{0}^{\infty}[g(t)]^{z} d t
$$

which is shorter than that of eq 9 for $z>1$. Starting at times of the order of or greater than $\tau_{L C M}$, the $L C$ will be mitigated to some extent and the coupling parameters $n_{p}$ can no longer maintain their shorter time constant value and will decrease with increasing time. In other words, as all chains are disentangling from each other to flow, the LC imposed on any chain are mitigated to some extent and consequently the coupling parameters $n_{p}$ will decrease with time. The Rouse rate slows down accordingly as $\tau_{p}^{-1}\left(\hat{\omega}_{c} t\right)^{-n_{p}(t)}$ where a time dependence has been introduced into the $n_{p}$. The correlation function which takes the LCM into account $\varphi_{p}^{L C M}(t)$, is obtained by solving

$$
d \varphi_{p}^{L C M} / d t=-\tau_{p}^{-1}\left(\hat{\omega}_{c} t\right)^{-n_{p}(t)} \varphi_{p}^{L C M}
$$

The solution is expected to follow closely $\varphi_{p}(t)$, the stretched exponential function with a constant $n_{p}$ given by eq 2 , for $t>\hat{t}_{c}$ until $t$ is of the order of and larger than $\tau_{L C M}$, after which $\varphi_{p}^{L C M}(t)$ decays faster than $\varphi_{p}(t)$. The dispersion of the terminal dispersion, represented by

$$
\left(<r(0) \cdot r(t)>/<r^{2}\right)_{L C M} \equiv g_{L C M}(t)=\left(8 / \pi^{2}\right) \sum_{p, o d d}\left(1 / p^{2}\right) \varphi_{p}^{L C M}(t) .
$$

in the time domain or by its Fourier transform in the frequency domain, is narrower than the corresponding function by eq 8 . The complex dynamic viscosity $\eta^{*}$ will be modified and the zero shear viscosity will be reduced. However, since $\varphi_{p}^{L C M}(t)$ tracks along with $\varphi_{p}(t)$ for times up to times of the order of $\tau_{p}^{*}$ (see insert in Figure 1), the molecular weight and time dependences of $\varphi_{p}^{L C M}(t)$ (or $g_{L C M}(t)$ ) remain the same as those of $\varphi_{p}(t)$ (or $g(t)$ ) with the same $n_{\mathrm{p}}$, which has already been given by eq 5 .

The $\mathrm{CM}$ at the present stage of development is at best a theoretical structure based on a physical principle [1-3] and as such does not provide a way to calculate the coupling parameter for any real system. Needless to say, the CM cannot predict in the present problem how exactly $n_{p}(t)$ changes with time. Nevertheless, the important point to be made here is that LCM and concomitant time-dependent $n_{p}(t)$ provide an explanation of the observed dielectric loss [36-40] of monodisperse polymer samples being narrower, particularly towards low frequencies, than predicted by eqs 2 and 8 with constant $n_{p}=0.4$ of the original CM [13-17]. This is possibly also true for the shear loss modulus peaks [16,21]. Experimental dielectric normal mode data can be used to infer $n_{p}(t)$. For example, parameterizing the time-dependence of $n_{p}(t), \varphi_{p}^{L C M}(t)$ can be solved numerically and used to fit the dielectric loss data. In the process the 
parameters introduced are determined. In fig 1 we show the dielectric loss function of frequency, $\varepsilon$ " $(\omega)$, calculated by taking the imaginary part of the integral [36],

$$
\varepsilon^{\prime \prime}(\omega) \propto \operatorname{Im} \int_{0}^{\infty}-[d<\vec{r}(0) \vec{r}(t)>/ d t] \exp (-i \omega t) d t,
$$

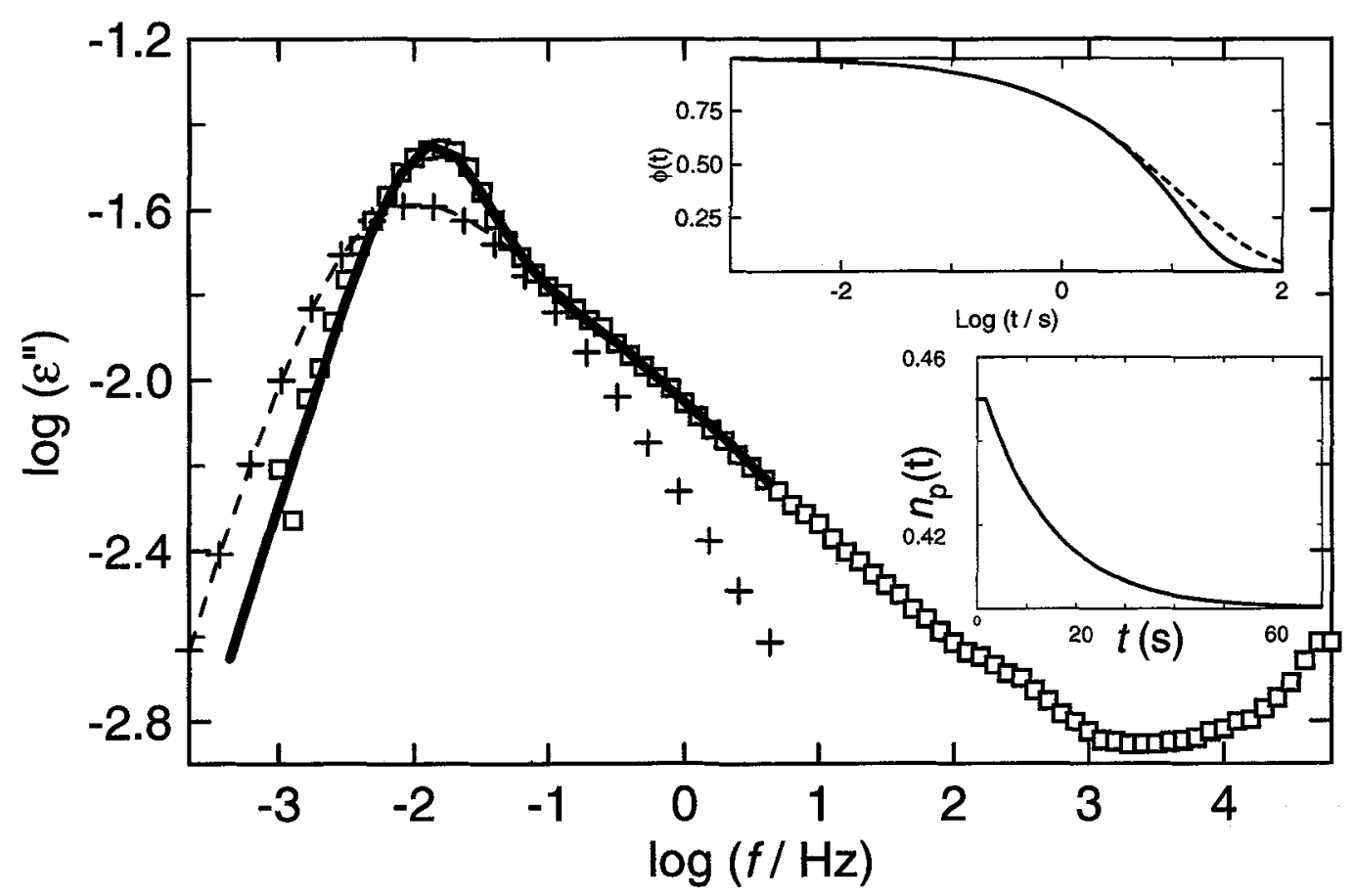

Figure 1. $\varepsilon$ " $(\omega)$ curves for monodisperse entangled linear PI melts calculated by the original CM for terminal relaxation with $n_{\mathrm{p}}=0.45$ summing over the first 4 modes (dashed curve) and only the first mode ( + ), and by the new CM that includes LCM with $n_{\mathrm{p}}(\mathrm{t})=0.45$ for $\mathrm{t}<\mathrm{t}_{\mathrm{i}}$ and $n_{p}(t)=\left(0.45-n_{\infty}\right) \exp \left(-\left(t-t_{i}\right) / \tau_{n}\right)+n_{\infty}$ for $t>t_{i}$ (solid curve). $\varepsilon$ "( $\left.\omega\right)$ data of monodisperse entangled linear PI melts with $M_{\mathrm{PI}}=140,000$ from Adachi et al. (squares). The lower inset shows the $n_{p}(t)$ used in the calculation for the new CM. The upper inset shows the relaxation functions for the original CM (dashed curve) and the new CM with LCM for $p=1$ mode only (solid curve).

where the end-to-end vector correlation function is given by eq $12, \varphi_{p}^{L C M}(t)$ obtained by integration of eq 11 and $n_{p}(t)$ is parametrized as $n_{p}(t)=0.45$ for $t<t_{i}$ and $n_{p}(t)=\left(0.45-n_{\infty}\right) \exp \left(-\left(t-t_{i}\right) / \tau_{n}\right)+n_{\infty}$ for $t>t_{i}$. In this parametrized form, $n_{p}(t)$ assumes the constant value of 0.45 until, when approaching the LCM time, it starts to decrease toward the smaller value of $n_{\infty}$. The initial value of $n_{p}(t)=n_{p}$ equal to 0.45 is chosen because it reproduces the $M^{3.6}$ dependence via eq 5 which remains valid even when LCM are present. The calculation performed with $n_{\infty}=0.38, t_{i}=0.15 \tau_{1}{ }^{*}, \tau_{n}=1.2 \tau_{1}{ }^{*}, \tau_{l}{ }^{*}=11 \mathrm{~s}$ and $\hat{t}_{c}=6.6 \times 10^{-5} \mathrm{~s}^{-1}$ provides a good fit to the $\varepsilon$ " $(\omega)$ data of high molecular weight monodisperse PI, shown by the thick solid line in fig 1. The value of $\tau_{1}$ of the $140,000 \mathrm{PI}$ at $T=273 \mathrm{~K}$ has been determined by Adachi and coworkers [36] to be $0.049 \mathrm{~s}$. The relation between $\tau_{L C M}$, and $\tau_{1}{ }^{*}$, given by $\tau_{L C M},=1.2 \tau_{1}{ }^{*}$, is consistent with eqs. 9 and 10.The result of the original CM without LCM calculated by eqs (8) and (13) with $n_{p}$ equal to 0.45 is shown by the dashed line in fig 1 and it is significantly broader in dispersion. Comparison with the result calculated with the inclusion of LCM indicates how mitigation of the lateral constraints sharpen the dielectric loss peak to a narrower full-width-at-half-height less than 1.5 decades, in agreement with experiment. These calculations have included four modes, $p=1,3,5$, and 7. For comparison, a calculation of the original CM including a single $p=1$ mode is also shown in fig $1(+)$. 
There is an apparent resemblance of lateral constraint mitigation (LCM) to contraint release (CR) introduced into the Doi-Edwards tube/reptation (T/R) model by Graessley [50]. However, the effect that LCM has in the CM is quite different from the effect of $C R$ in the T/R model. CR results from the reptation of the neighboring chains that provide the tube constraints. It was proposed to reduce $\eta$ and increase $J_{\varepsilon}^{0}$ predicted by the $T / R$ model for monodisperse systems and to bring them into better agreement with experiment. Pure reptation predicts $\eta \propto M^{3.0}$ and although $\mathrm{CR}$ lowers the predicted values of viscosity it does not alter the exponent. As explained before, LCM does not change the $M^{2 /\left(1-n_{1}\right)} \approx M^{3.4}$ molecular dependence of $\tau_{p}^{*}$. in the original CM either. However, as we shall see LCM in the $\mathrm{CM}$ and $\mathrm{CR}$ in the T/R model predict different behaviors of dilute polyisoprene probe chains in very high molecular weight linear polybutadiene matrices and in loosely cross-linked polybutadiene matrices. These differences will be discussed in the next section.

\subsection{Dilute polyisoprene probes in polybutadiene matrices}

The inclusion of LCM in the terminal relaxation of monodisperse polymer melts improves the comparison of the shape of the dielectric loss peak of PI predicted by the new CM with experimental data. This is only a theoretical result which lacks an exact prescription of how the shape of a monodisperse entangled PI melt can be calculated from first principles. Thus, it would be hard to evaluate the merit of this new formulation of the $\mathrm{CM}$ for homopolymer melts had this been the only result that follows as a consequence. Fortunately, there are other immediate consequences that provides additional critical tests of the new CM. If LCM is important for a monodisperse homopolymer, then dilute amount of PI probes introduced in a matrix of a very high molecular weight PB or in a crosslinked PB matrix (henceforth these systems are referred to as $\mathrm{PI} / h \mathrm{~PB}$ and $\mathrm{PI} / x \mathrm{~PB}$ respectively) will have their LC permanently in place for all times, as far as relaxation of the end-to-end vector of the guest PI chain is concerned. The coupling parameters $n_{p}$ of the guest PI should remain constant throughout because the LC imposed on the PI chain by the long host PB chains are not mitigated. The LCM of the PI probe having been suppressed in PI/hPB and in $\mathrm{PI} / x \mathrm{~PB}$, the result of the original $\mathrm{CM}$ calculated by eq 13 with the end-to-end vector correlation function given by eq 8 with constant $n_{p}$ will be recaptured. Stated differently, the new CM affords another prediction: the $\varepsilon$ " $(\omega)$ peaks of PI in PI/hPB and in PI $x$ PB will become broader than in monodisperse PI homopolymer melt. Verification of this additional prediction of the new CM should considerably enhance the credibility of the model.

The experimental measurements of dielectric relaxation of a small amount $(-5 \mathrm{wt} . \%)$ of probe PI with molecular weight $M_{\mathrm{PI}}$ in PB of different molecular weights, $M_{\mathrm{PB}}$, have been reported recently by Adachi and coworkers [41] and by Watanabe and coworkers [42]. The experimental data of a $27 \mathrm{~K}$ PI in $202 \mathrm{~K}$ PB by Adachi et al. (filled triangles) are shown in fig 2. Both groups have found for sufficiently long PI guest chains which entangle with the host PB chains that the loss curves $\varepsilon$ " $(\omega)$ broadens with increasing $M_{\mathrm{PB}}$. The broadening is prominent especially in the high frequency side of the peak and appears to occur also on the low frequency side of the peak, although there is limited amount of dielectric loss data on the low frequency side due to the presence of conductivity contributions. In a fixed high molecular weight PB matrix, the dielectric relaxation times of PI were determined as $1 /\left(2 \pi f_{\max }\right)$ from the loss maximum frequencies $f_{\max }$. After corrected for the differences in monomeric friction of the probe PI chains in the blends, the dielectric relaxation times, $\tau_{\max }$, were found to have the $M^{3.0}$ dependence by Adachi et al. and a slightly larger $M$-dependence by Watanabe et al. in blends formed by PB with $M_{\mathrm{PB}}=152 \mathrm{~K}$. Via eq 5 , we infer $n_{\mathrm{p}}=0.33$ from the $M^{3.0}$ dependence of $\tau_{\max }$. If LCM were totally suppressed in the PI $h$ PB studied by Adachi et al and Watanabe et al., the loss curves $\varepsilon$ "' $(\omega)$ are calculated according to eqs. (5), (8) and (13) of the original $\mathrm{CM}$ without lateral constraint mitigation so that $n_{\mathrm{p}}=0.33$ is time independent. In fig 2 the calculated sum of the contributions of the odd modes, $p=1,3,5$ and 7 to $\varepsilon$ " $(\omega)$ is shown, and it fits the experimental data well. The dispersion of the $27 \mathrm{~K}-\mathrm{PI} / 202 \mathrm{~K}-\mathrm{PB}$ data is slightly broader than that of the 
$49 \mathrm{~K}-\mathrm{PI} / 89 \mathrm{~K}-\mathrm{PB}$. This trend is consistent with the ratio $M_{\mathrm{PB}} / M_{\mathrm{PI}}$ being larger and the suppression of LCM being more complete in the former than in the latter.

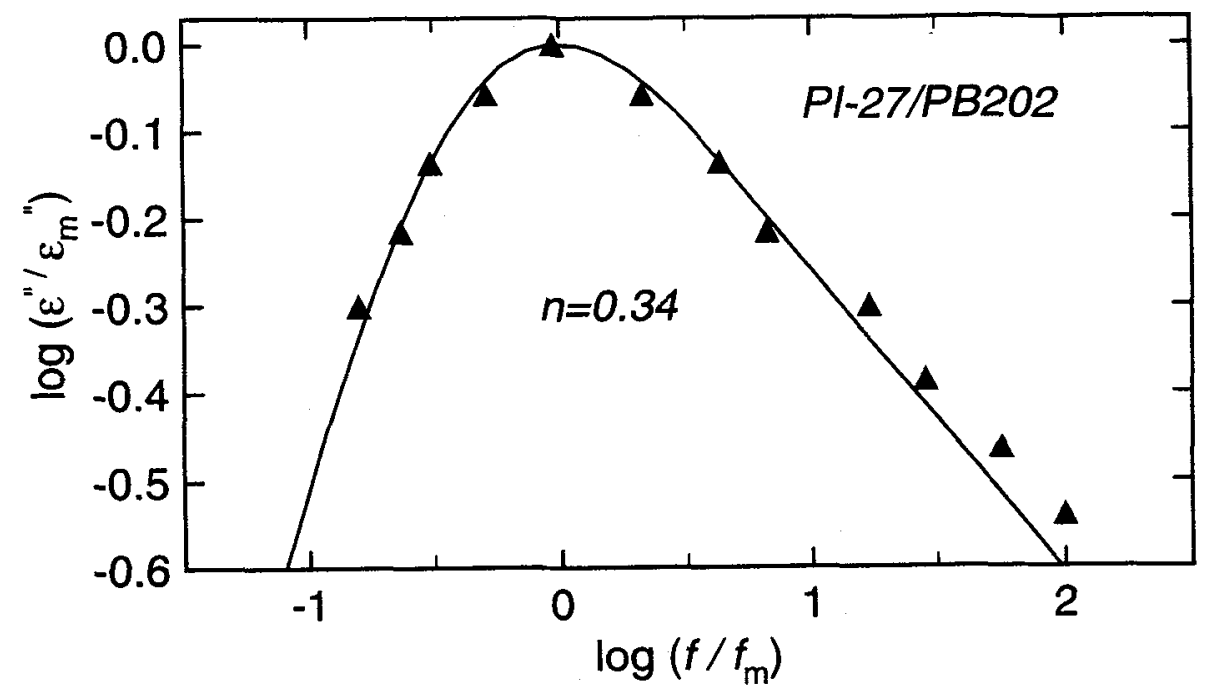

Figure 2. Normalized $\varepsilon^{\prime \prime}(\omega)$ data of (a) PI with $M_{\mathrm{Pl}}=27,000$ in a very high molecular weight $\left(M_{\mathrm{PB}}=202,000\right)$ PB matrix (solid triangles) from Adachi et al. (Ref.43). Normalized $\varepsilon^{\prime \prime}(\omega)$ curve calculated with $n_{\mathrm{p}}=0.34$ by the CM.

Adachi and coworkers studied the dielectric normal mode relaxation of PI trapped in crosslinked natural rubber networks [52] and in PB networks [43]. The experimental data were reviewed by Adachi and Kotaka [36,53]. The PB networks used were loosely crosslinked with the molecular weight between crosslinks $M_{\mathrm{x}}>M_{\mathrm{e}}$, where $M_{e}$ is the molecular weight between entanglements. The dielectric relaxation

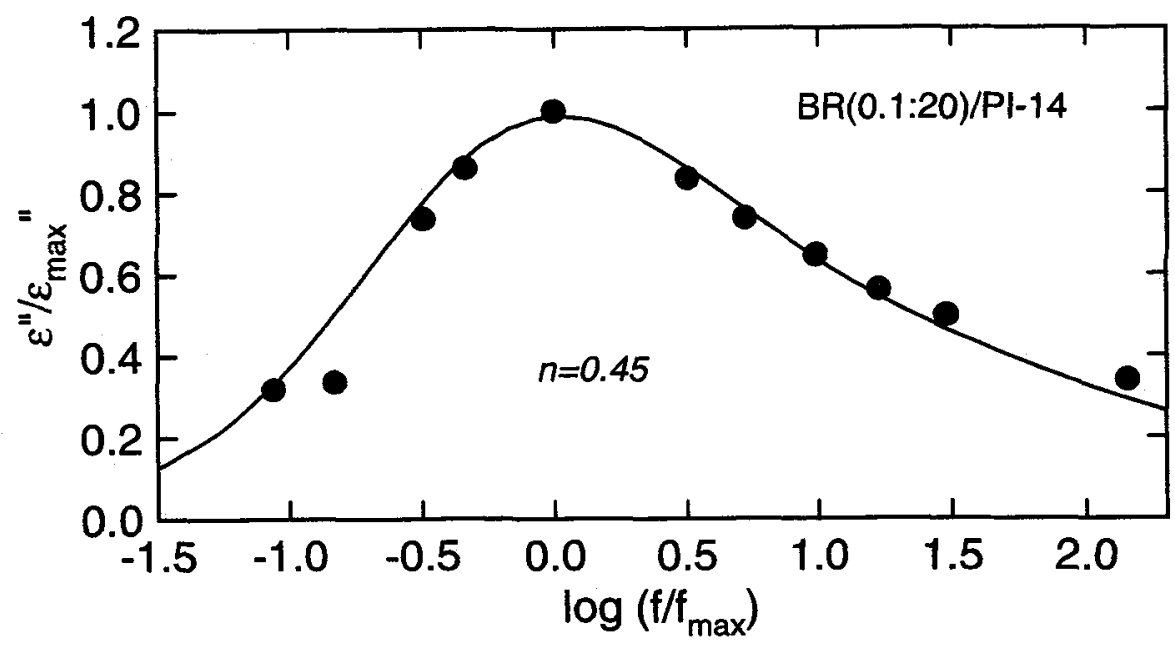

Figure 3. Normalized $\varepsilon$ " $(\omega)$ data of free PI chains with $M_{\mathrm{PI}}=13,500$ trapped in polybutadiene matrix with $M_{\mathrm{x}}=11,000$ (solid circles). Normalized $\varepsilon "(\omega)$ curve calculated with $n_{\mathrm{p}}=0.45$ by the $\mathrm{CM}$. 
time $\tau_{\max }$ of $\mathrm{PI}$ in $\mathrm{PI} / \mathrm{xPB}$, after correction to iso-friction state has been made, has the $\left(M_{\mathrm{PI}}\right)^{3.6}$ dependence. The loss curve $\varepsilon$ " $(\omega)$ is broader than that of pure bulk monodisperse entangled PI. The experimental data of 14K-PI trapped in a PB network labelled BR(90.1:20) are shown in fig 3 (filled circles). In the same figure the solid curve represents the theoretical $\varepsilon$ " $(\omega)$ which is the sum of the contributions from the odd modes $p=1,3$, and 5 . The calculation proceeds in the same manner as discussed in the previous paragraph for PI/hPB blends, i.e. via eqs (5), (8) and (13) except $n_{p}=0.45$ in the present case. This value of $n_{\mathrm{p}}$ is determined from eq 5 by the observed $\left(M_{\mathrm{PI}}\right)^{3.6}$ dependence of the dielectric relaxation time. By inspection, fig 3 indicates that there is good agreement between the theoretical and the experimental $\varepsilon$ " $(\omega)$, confirming that the observed broad dispersion is consistent with the new CM with LCM being suppressed in a network.

Consider first entangled concentrated PI solutions and melts. The tube/reptation model with or without configuration-independent constraint release (CICR) [42,50,54] or configuration-dependent constraint release (CDCR) [55] is still unsatisfactory in explaining the frequency dependence of dielectric loss $\varepsilon "(\omega)$. The shape of the $\log \left(\varepsilon "(\omega) / \varepsilon{ }^{\prime}{ }_{\max }\right)$ versus $\log \left(f / f_{\max }\right)$ curve is broader than the $T / R$ model without CICR or CDCR, especially in the high frequency tail where $\varepsilon " \propto f^{\mathrm{m}}$ with $m \approx 0.30-0.33$. CICR model was found to be qualitatively different from the dielectric data of PI in various PB matrices [56]. The CDCR model well describes $\varepsilon$ " $(\omega)$ for $\omega \leq 10 \omega_{\max }$ but underestimates the higher frequency dielectric loss tail by predicting that $\varepsilon^{\prime \prime} \propto f^{1 / 2}$, which lies below the experimental $\varepsilon^{\prime \prime}(\omega)$ data of PI melts and solutions and PI in various PB matrices $[56,57]$. The predicted $M^{3.0}$ molecular weight dependence of the relaxation time $\tau_{\max } \equiv 1 /\left(2 \pi f_{\max }\right)$ with or without constraint release is also at variance with the observed $M^{3.6-3.7}$ dependence. In principle, inclusion of contour length fluctuations proposed by Doi into the T/R model can have a molecular weight dependence that resembles $M^{3.4}$ dependence in some molecular weight range. However, Adachi and coworkers have ruled out the contour length fluctuation mechanism in finding a $M^{3}$ dependent relaxation time of the probe PI chains in matrices with extremely high molecular weight PI $[36,53,57,58]$ and $P B$ chains.

The original application of $\mathrm{CM}$ to the terminal relaxation proposed over ten years ago with a constant coupling parameter in the range $0.40 \leq n_{\mathrm{p}} \leq 0.45$ to explain the $M^{3.4-3.7}$ dependence of viscosity via eq 5 cannot explain the frequency dependence of the dielectric loss curves of PI melts and solutions either. The calculated $\varepsilon^{\prime \prime}(\omega)$ is too broad on the low frequency side of the peak, and also on the high frequency side but only in the vicinity of the peak (see fig 1 ). Although, the $f^{\mathrm{m}}$-dependence with $m \approx 0.30-0.33$ of the high frequency tail of $\varepsilon$ " can be well explained by the original $\mathrm{CM}$ when all odd modes are included. The discrepancy of the original $\mathrm{CM}$ with experimental data could be described as if, on approaching the peak from the high frequency side, another mechanism starts to take over to reduce the dispersion resulting in a narrower loss peak. We have come to realize that this mechanism is the mitigation of the lateral constraints which must come into play at times of the order of the terminal relaxation time $\left(\approx \tau_{\max } \equiv 1 /\left(2 \pi f_{\max }\right)\right)$ because the end-to-end vectors of all chains are relaxed at about this time. Lateral constraints (LC) were not emphasized and their eventual mitigation (LCM) was not considered in the original CM [13-17] which has a time-independent coupling parameter $n_{\mathrm{p}}$. The new CM incorporates LCM which makes $n_{\mathrm{p}}$ time-dependent, decreasing with time starting in the vicinity of the terminal relaxation time and beyond. As shown in fig 1, for reasonable choice of the time-dependence of $n_{\mathrm{p}}$ the entire frequency dependence of $\varepsilon$ " $(\omega)$ observed in concentrated solutions and melts of PI can be reproduced. The predicted $M$ and $T$-dependences of $\tau_{\max }$ of the new CM remains the same as the original $\mathrm{CM}$ according to eq 5, where $n_{\mathrm{p}}$ appearing therein is now the initial constant coupling parameter before it starts to decrease due to the onset of LCM. The recognition of the importance of LC in the new CM brings it in harmony with earlier propositions [44-46], simulations [31-33] and conclusions made from recent experimental investigations [59] that motion of an entangled chain is more facile along the general directions defined by the LC. However, the chain motion in the new CM is governed by the general principle of contraint dynamics modified by LCM. The motion of the chain is slowed down by the LC according to the prescription of the CM, but this slowed motion is still a better option for the chain than resorting to the most difficult motion of all, i.e. diffusion along its own contour or reptation. In other 
words, the presence of LC or even the existence of a tube of diameter as large as 3-5 nm does not necessary mean that the chain has to reptate. Forcing the chain to reptate leads to an untenable physical situation that cannot be made consistent with various experimental facts even if additional mechanisms such as constraint release and contour length fluctuation are augmented [36-43,53,54]. As we have shown, there is a better option given by the CM with inclusion of LCM.

\section{Conclusion}

Dynamics in polymers occur over an immense range of length scales from very local motions to motion of the entire chain. Mechanical spectroscopy (viscoelasticity) is the experimental tool that has a direct connection to practical problems in the application of polymeric materials. Many interesting physical phenomena have been discovered and there are plenty of opportunities for new effects to be discovered in this immense field where challenging problems abound. Molecular units are densely packed together and an understanding of their complicated motions require solutions of "many-body" problems of different length-scales in several viscoelastic zones. In this paper we have pointed out some of the outstanding and formidable problems in viscoelasticity of polymers that defy solution by many well known theoretical treatments. Remarkably, the coupling model has provided viable solutions to these problems as well as those in other fields. This is perhaps not surprising, because the coupling model is based on a physical principle that is generally applicable to densely packed interacting systems.

\section{References}

[1] Ngai K.L., Comment Solid State Phys. 9 (1979) 127; Ngai K.L. and White C.T., Phys.Rev. B20 (1979) 2475.

[2] Ngai K.L. and Tsang K.Y., Macromolec.Symp., 90 (1995) 95; Ngai K.L., Peng S.L. and Tsang K.Y., Physica A191 (1993) 523; Ngai K.L. and Rendell R.W., J. Non-Cryst.Solids 131-133 (1991) 233;

Tsang K.Y. and Ngai K.L., Phys.Rev.E, Rapid Commun. (1996) in press.

[3] Rendell R.W., Phys. Rev. E48 (1993) R17; Rendell R.W., J. Appl. Phys. 75 (1993) 7626.

[4] Ngai K.L., in "Relaxational Processes in Disordered Systems", Richert R. and Blumen A. Eds., (Springer-Verlag, Berlin, 1993) p.89.

[5] For applications to polymer viscoelasticity, see Ngai K.L. and Plazek D.J., Rubber Chem. Tech. Rubber Review 68 (1995) 376.

[6] MacKay R.S. and Meiss J.D., "Hamiltonian Dynamical Systems" (Adam Hilger, Bristol, 1987).

[7] Colmenero J., Arbe A. and Alegria A., Phys.Rev.Lett. 71 (1993) 2603; J. Non-Cryst.Solids 172-174 (1994) 126; Ngai K.L., Colmenero J., Arbe A., and Alegria A., Macromolecules 25 (1992) 6727.

[8] Zorn R., Arbe A., Colmenero J., Frick B., Richter D., and Buchenau U., Phys. Rev. E52 (1995) 781.

[9] Cramer C., Funke K. and Saatkamp T., Philos. Mag. B71 (1995) 701.

[10] Cramer C., Funke K., Buscher M., and Happe A., Philos. Mag. B71 (1995) 713.

[11] Ngai K.L., Cramer C., Saatkamp T., and Funke K., in Proceedings of Workshop on Non-Equilibrium in Supercooled Fluids, Glasses and Amorphous Materials, 25-29 September 1995, Pisa, Italy, D. Leporini, Ed. 1996 (World Scientific, Singapore, in press).

[12] Lewis L.J. and Wahnström G., Phys. Rev. E50 (1994) 3865; Roland C.M., Ngai K.L. and Lewis L.J., J. Chem. Phys. 103 (1995) 4632.

[13] Ngai K.L. and Rendell R.W., Polym. Prepr. Am. Chem. Soc. Div. Polym.Chem. 23 (1982) 46.

[14] Ngai K.L. and Plazek D.J., J. Polym. Sci. Poly. Phys. Ed. 23 (1985) 2159.

[15] Ngai K.L. and Plazek D.J., J. Polym. Sci. Poly, Phys. Ed. 24 (1986) 619.

[16] McKenna G.B., Ngai K.L. and Plazek D.J., Polymer 26 (1985) 1651.

[17] Rendell R.W., Ngai K.L. and McKenna G.B., Macromolecules 20 (1987) 2250.

[18] Kohlrausch R., Pogg. Ann. Phys. 12(3) (1847) 393.

[19] Plazek D.J. and Ngai K.L., Macromolecules 24 (1991) 1222; Boehmer R., Ngai K.L., Angell C.A., 
and Plazek D.J., J. Chem. Phys. 99 (1993) 4201.

[20] Ngai K.L., Echeverria I. and Plazek D.J., Macromolecules, submitted.

[21] Plazek D.J., Colloid Polym. Sci. 272 (1994) 1430; D.J.Plazek and V.M. O'Rourke, J. Polym.Sci., A2,9 (1971) 209; Gray R.W., Harrison, B., and Lamb J., Proc.R.Soc.Lond. A356 (1977) 77.

[22] Ferry D.J., "Viscoelastic Properties of Polymers" 3rd ed. (J.Wiley, New York, 1980).

[23] Plazek D.J., J. Polym. Sci. A-2 (1968) 621.

[24] Cavaille J.-Y., Jordan C., Perez J., Monnerie L. and Johari G., J. Polym. Sci.:Part B, Polym. Phys. 25 (1987) 1235.

[25] Palade L.I., Verney V. and Attené P., Macromolecules 28 (1995) 7051.

[26] Plazek D.J., Zheng X.D. and Ngai K.L., Macromolecules 25 (1992) 4920.

[27] Plazek D.J., Chay I.C., Ngai K.L. and Roland C.M., Macromolecules 28 (1995) 6432.

[28] Cochrane J., Harrison, G., Lamb, J. and Phillips D.W., Polymer 21 (1980) 837; Ngai K.L., Schönhals A. and Schlosser E., Macromolecules 25 (1992) 4519.

[29] Plazek D.J., Schlosser E., Schönhals A. and Ngai K.L., J.Chem. Phys. 98 (1993) 6488.

[30] Ngai K.L., Plazek D.J. and Bero C., Macromolecules 26 (1993) 1065.

[31] Skolnick J., Kolinski A.,Adv. Chem. Phys.78(1990)223; Ngai K.,Macromolecules 24 (1991) 1561.

[32] Pakula, T.and Geyler S., Macromolecules 20 (1987) 2909; 21 (1988) 1665.

[33] Kremer K.and Grest G., J.Chem.Phys. 92 (1990) 5057;Ngai K.L., Macromolecules 25 (1992) 2184.

[34] Raju V.R., Rachapudy H., and Graessley W.W., J. Polym. Sci. Poly. Phys. Ed. 17 (1979) 1223.

[35] Bero C.A.and Roland C.M., Macromolecules 29 (1996) 1562 and references therein.

[36] For a review see Adachi K.and Kotaka T., Prog. Polym. Sci. 18 (1993) 585.

[37] Adachi K., Yoshida H., Fukui F., and Kotaka T., Macromolecules 23 (1990) 3138.

[38] Boese D., Kremer F., and Fetters L.J., Macromolecules 23 (1990) 829 ; ibid. 23 (1990) 1826.

[39] Yoshida H., Watanabe H., Adachi K., and Kotaka T., Macromolecules 24 (1991) 2981.

[40] Bero C. and Roland C.M., Macromolecules, in press.

[41] Adachi K., Wada T., Kawamoto T., and Kotaka T., Macromolecules 28 (1995) 3588.

[42] Watanabe H., Urakawa O., Yamada H. and Yao M.-L., Macromolecules 29 (1996) 755.

[43] Adachi K., Nakamoto T., and Kotaka T., Macromolecules 22 (1989) 3111.

[44] “...] branched polymer may be more effectively trapped by its entanglement [than a linear polymer] because its various arms do not allow a simple snaking motion through the entanglements", Long V.C., Berry G.C.; and Hobbs L.M., Polymer 5 (1964) 517.

[45] "The viscous flow in polymers involve more motion parallel to the chain backbone than do retarded elastic conformational changes.", Plazek D.J., J. Phys. Chem. 69 (1965) 3480.

[46] Bueche F., J. Chem. Phys. 22 (1954) 603.

[47] DeGennes P.G., J. Chem. Phys. 23 (1980) 829.

[48] Doi M., and Edwards S.F., The Theory of Polymer Dynamics (Clarendon Press, Oxford, 1986).

[49] Butera R., Fetters L.J., Huang J.S., Richter D., Pyckhout-Hintzen W., Zirkel A., Farago B., and Ewen B., Phys. Rev. Letters 66 (1991) 2088.

[50] Graessley W.W., Adv. Polym. Sci. 16 (1974) 1.

[51] Ngai K.L., Roland C.M., and Greaves G.N., J.Non-Cryst.Solids 182 (1995) 172.

[52] Poh B.T., Adachi K., and Kotaka T., Macromolecules 20 (1987) 2563.

[53] Adachi K. and Kotaka T., Polymer Yearbook 6 (1990) 43.

[54] Watanabe H. and Kotaka T., Chemtracts-Macromolecular Chemistry 2 (1991) 139.

[55] Watanabe H. and Tirrell M., Macromolecules 22 (1989) 927.

[56] Watanabe H., Yamazaki M., Yoshida H., and Kotaka T., Macromolecules 24 (1991) 5372.

[57] Kotaka T. and Adachi K., Macromol. Chem. Macromol. Symp. 79 (1994) 125; Proc. IUPAC Microsymp., 33rd (1993).

[58] Adachi K., Nishi I., Doi H., and Kotaka T., Macromolecules 24 (1991) 5843.

[59] Russell T.P. et al., Nature 365 (1993) 235. 